\title{
Médiévales
}

Langues, Textes, Histoire

\section{Julia Gonnella, Friederike Weis, Christoph Rauch (éd.), The Diez Album, Contexts and Contents}

Leyde, Brill («Islamic Manuscripts and Books », 11), 2016, XVIII-672 p.

\section{Anna Caiozzo}

\section{(2) OpenEdition}

\section{Journals}

Édition électronique

URL : https://journals.openedition.org/medievales/11167

DOI : 10.4000/medievales. 11167

ISSN : $1777-5892$

Éditeur

Presses universitaires de Vincennes

Édition imprimée

Date de publication : 28 décembre 2020

Pagination : 232-236

ISBN : 978-2-37924-146-8

ISSN : 0751-2708

Référence électronique

Anna Caiozzo, « Julia Gonnella, Friederike Weis, Christoph Rauch (éd.), The Diez Album, Contexts and Contents », Médiévales [En ligne], 79 | automne 2020, mis en ligne le 28 janvier 2021, consulté le 07 janvier 2023. URL : http://journals.openedition.org/medievales/11167 ; DOI : https://doi.org/10.4000/ medievales. 11167

Ce document a été généré automatiquement le 7 janvier 2023

Tous droits réservés 


\section{Julia Gonnella, Friederike Weis, Christoph Rauch (éd.), The Diez Album, Contexts and Contents}

Leyde, Brill (« Islamic Manuscripts and Books », 11), 2016, XVIII-672 p.

\section{Anna Caiozzo}

\section{RÉFÉRENCE}

Julia Gonnella, Friederike Weis, Christoph Rauch (éd.), The Diez Album, Contexts and Contents, Leyde, Brill («Islamic Manuscripts and Books », 11), 2016, XVIII-672 p.

1 L'ouvrage est un collectif dédié à la mémoire de Ernst J. Grube (m. 2011), qui réunit vingt-et-une contributions portant sur les célèbres Albums Diez de Berlin bien connus des orientalistes et des historiens de l'art, mais sur lesquels les études demeurent rares. Ces cinq albums cotés Diez 70 à 74 portent le nom du diplomate prussien Heinrich Friedrich von Diez (1751-1817) qui les rapporta de Constantinople où il était en poste auprès de la Sublime Porte.

2 En juin 2013, deux conservatrices du Musée de Berlin, Julia Gonnella et Friederike Weis, et un conservateur de la Staatsbibliothek, Christopher Rauch, ont organisé un colloque d'importance réunissant nombre de chercheurs, en particulier ceux ayant travaillé sur les homologues de ces albums et certaines de leurs sources, les Albums Hazine du Topkapi Sarayi à Istanbul qui, quant à eux, avaient fait l'objet en 1980 d'une rencontre à Londres sous l'égide de Ernst J. Grube.

3 Les Albums Diez sont sortis de l'ombre assez tardivement; l'une des toute premières études fut celle de Ernst Kühnel en 1959, suivie de celle de Mazhar şevket Ipsiroglu ${ }^{1}$. Deux autres avaient apporté un regain d'intérêt : celle de David Roxburgh en 1995, dans la revue Muqarnas et celle de Karine Rührdanz, qui avait analysé les folios d'époque ilkhanide et montré leur origine probable dans l'illustration d'une copie de l'Histoire universelle de Rashīd al-dīn². 
4 Les Albums Diez sont, dans les faits, quatre compendiums de matériel disparate, principalement iconographiques, comportant quatre cents illustrations d'époques différentes, principalement des $\mathrm{XIV}^{\mathrm{e}}$ et $\mathrm{XV}^{\mathrm{e}}$ siècles (ilkhanide, djalayride, timouride), mais incluant aussi des matériaux ottomans, chinois, européens. Les trois premiers sont demeurés dans l'état dans lequel ils avaient intégré les collections allemandes depuis leur constitution et acquisition en Turquie au xviII ${ }^{e}$ siècle, mais les deux autres, postérieurement composés, rassemblent des folios épars.

5 Du point de vue de l'histoire culturelle, les Albums Diez représentent une dimension de l'orientalisme, et principalement de l'orientalisme allemand et de son intérêt pour l'histoire de l'art des mondes orientaux islamisés, car les albums étaient une pratique connue depuis l'époque timouride et safavide; ils constituaient des sources précieuses pour la connaissance de la peinture, des méthodes employées par les peintres et miniaturistes du passé par la constitution de modèles issus d'horizons différents, ou encore d'essais préparatoires, ou même de collectes de modèles émanant de grands peintres qui se transmettaient d'atelier en atelier, d'une époque sur l'autre, et qui illustrent bien les propos du peintre safavide Dust Muhammad sur ses prédécesseurs et leur talent ${ }^{3}$.

6 D'un point de vue plus pragmatique, ces albums constitués en Orient sous les Ottomans sont le produit d'un temps, d'une époque, de choix volontaires opérés pour réunir des exemplaires de peintures, de sujets, parfois en pillant, découpant des manuscrits existants comme le montre, entre autres tragédies, l'histoire du Grand Shāh nāma mongol, de collectes opérées à l'occasion de conquêtes dans diverses œuvres que les chercheurs essaient d'identifier ${ }^{4}$, voire dans d'autres albums comme ceux d'Istanbul.

Et, de fait, le destin des Albums Diez les associe étroitement à celui des Albums Hazine. En effet, comme l'ont montré diverses études, les Albums Hazine 2152 et 2153 ou encore 2160 , sans compter le B 411, rassemblant du matériel d'époque timouride et turkmène, furent des sources de choix dans lesquels puisa le commanditaire turc des Albums Diez. Contrairement aux Albums Hazine, les Albums Diez sortirent tardivement de l'ombre, grâce aux grandes expositions internationales, puis en 2013, la Staatsbibliothek les fit numériser et les offrit en consultation aux lecteurs depuis sa base numérique.

On doit, dans un premier temps, rendre hommage aux organisateurs du colloque et aux éditeurs, devant la qualité d'une majorité de contributions et leur volonté de lever le voile à la fois sur les intentions qui présidèrent à la constitution des albums et sur le matériel qui s'y trouve conservé comme sur l'usage que l'historien de l'art pourrait en faire.

9 Le volume est organisé en six parties thématiques. La première partie est essentielle, puisqu'elle resitue l'histoire contextualisée des albums. Julian Raby, par exemple, consacre son analyse à leur genèse à partir des Albums Hazine, et à l'esprit qui présida à cette " création ». Il émet l'hypothèse que ce fut cet eunuque chassé du palais en 1789 par Sélim III, qui avait orchestré et leur composition et leur vente dans les années 1789, notamment à partir des Albums Hazine 2152 et 2153 incluant des peintures ou des dessins remarquables à l'image de ceux du grand peintre timouride Muhammad-i Khayyām. Ce point est repris plus loin par Lâle Uluç, qui s'interroge sur l'usage de ces Albums, H. 2153 et H. 2160 notamment marqués de la tughra de Selim I ${ }^{\text {er }}$ (r. 1512-20) et où sont conservées des calligraphies célèbres (celles du prince timouride Baysunghur Mirza, par exemple). Ces Albums Hazine, dont Dust Muhammad écrivait qu'ils étaient 
faits pour le regard et l'agrément des connaisseurs, n'inspirèrent cependant pas les illustrations des manuscrits ottomans.

Quant à lui, David J. Roxburgh insiste sur la personnalité du comte von Diez et son lien éventuel dans la composition de ses albums. Il retrace le parcours de ce bourgeois qui, par ses compétences d'orientaliste, devint diplomate sous Frédéric Guillaume II à la fin du XVIII ${ }^{e}$ siècle. Heinrich Friedrich von Diez avait une passion pour la littérature orientale, persane notamment. Au cours de sa vie, il avait mûri un grand projet intellectuel: montrer les points de convergence ou de divergence de la conscience humaine en Orient et Occident en analysant les éléments linguistiques (grec, latin, langues orientales) et les traditions culturelles. Aussi, s'interroger sur la part de von Diez dans la composition des albums était légitime, puisque les albums furent composés pour satisfaire ses centres d'intérêt. On comprend alors que les albums illustraient, par touche, les grandes œuvres de la littérature persane qu'il affectionnait: Shāh nāma, Kalìla et Dimna, Humayūn nāma, etc. Christoph Rauch complète le propos sur la passion orientaliste de von Diez qui rêvait de traduire la Bible en turc comme il avait traduit l'épopée des Turcs, et dont le legs de dix-sept mille volumes à la Bibliothèque royale en 1817 ne retient pas l'attention des conservateurs de l'époque sur le matériel illustré, car les manuscrits enluminés ne firent l'objet d'un intérêt spécifique que de façon très tardive, en France d'ailleurs comme en Allemagne.

11 Enfin, Zeren Tanindi tente d'expliquer le rôle des répétitions d'illustrations dans les deux types d'albums, liées selon elle à des choix des différents maîtres les ayant possédés, mais surtout à une volonté de montrer les collectes de modèles exemplaires ou représentant des exercices de style en introduisant des variations de modèles. Dans un esprit semblable, Simon Rettig analyse les travaux du bibliothécaire en chef de l'atelier de Baysunghur, Ja far Tabrizì dans l'album Diez A fol. 74 et Diez A fol. 70. Il suit donc l'évolution de l'écriture depuis l'époque djalayride, où ce peintre fit ses débuts, jusqu'à l'époque timouride où un album comme le B 411 est véritablement promu album de modèles remarquables.

Cette partie préliminaire sur le concept délicat et complexe de la définition d'un album et de son objectif doit retenir l'intérêt principal du lecteur, qui pourra ensuite survoler la deuxième partie, "The Albums' Contents : From the Mongols to the Timurids ", bien explorée antérieurement et par ailleurs.

13 Certes, pour les historiens de l'art, le fleuron des Albums Diez, outre les très belles miniatures d'époque djalayride et timouride, reste les scènes dédiées à la vie de cour des Mongols, sur lesquelles Karine Rührdanz avait donné un point de vue très éclairant. Charles Melville y apporte sa lecture, en rappelant très justement que ces miniatures rendent hommage au mode de vie des Mongols antérieur à l'arrivée en Iran, avec la valorisation de la chasse ou des paysages naturels qu'ils affectionnaient, et surtout via les scènes militaires, ou encore de majesté, que Yuka Kadoi répartit en trois types. C. Melville rappelle le protocole d'intronisation que les miniatures ne reproduisent pas et décrit les fonctions des grands officiers, dont le rôle du keshiq, qu'il a bien étudié. Parmi les autres contributions, celle de Claude-Peter Haase rappelle que les concepts apportés par les Mongols dans les terres islamisées sont novateurs en matière de symboles. Il faut quand même souligner que les Saldjoukides ont remployé des formes d'insignes iraniens avant que les Mongols ne les adoptent. L'iconographie peut parfois être instructive quant aux pratiques culturelles ou sociales, comme le montre Barbara Brend: elle observe comment deux pratiques picturales coexistent dans les Albums 
Diez, celle relative à la l'observation de la vie des Turkmènes et de leurs chevaux, l'autre sur les façons de peindre les animaux, inspirées des modèles chinois qui triomphent dans la peinture d'époque djalayride. De même, Filiz Cakir Philipp rappelle que le rôle des manuscrits enluminés était d'exalter la nation mongole dans son rôle de conquérante et dans ses éléments identitaires, mais aussi de s'instituer comme héritière des grands héros et guerriers de l'Iran ancien que l'épopée des rois de Perse promouvait.

14 La troisième partie ("The Albums' Contents: Drawings and Sketches») réunit un ensemble de contributions techniques sur les modèles des Albums Diez et le rôle que les calligraphes et les peintres leur attribuaient, comme l'explique Yves Porter. On peut se rappeler, comme le décrit par ailleurs l'Arzadasht de Ja'far Tabrizī, la façon pratique dont fonctionnaient les ateliers d'époque timouride, à la fois par division des tâches, spécialisation et reproduction de modèles quasi figés, $\mathrm{y}$ compris dans la répétition de composition des scènes illustrant de nombreux manuscrits appartenant à des corpus identiques ou différents, pour des situations similaires ou des scènes identiques. Certes, des exceptions existent, car l'un des dessins des plus célèbres et des plus atypiques des Albums Diez, analysé par Friederike Weis, est une copie d'antique. F. Weis étudie les dessins de ce peintre timouride œuvrant au sein de l'atelier de Baysunghur et auquel on attribue la copie d'un camée d'époque hellénistique, réalisé à Alexandrie et qui parvint à Hérat via les conquêtes de Tamerlan. Après bien des vicissitudes, le camée fut acquis par le prince Farnèse puis par le musée archéologique de Naples. D'ailleurs, pour relayer les réserves de F. Weis sur les dessins aux encres carbones, Oliver Hahn propose d'utiliser des méthodes d'investigation non invasives, prenant pour sujet d'expérimentation, le magnifique cloud collar du Diez A fol. 73, p. 54.

Un des chapitres les plus enthousiasmants de cet ouvrage demeure sans aucun doute « Repatriations : the Diez Albums as a Source for Reconstructing Lost Art ». En effet, les Albums Diez comme leurs homologues Hazine sont des sortes de cavernes d'Ali Baba pour l'historien de l'art en quête d'informations sur les manuscrits perdus ou dispersés. Ainsi peut-on supposer que certaines des miniatures des Albums Diez font partie intégrante d'un des plus tristement célèbres exemplaires du Livre des rois de Firdawsī, le grand Shāh nāma mongol, sur lequel revient Robert Hillenbrand après le travail de doctorat d'un de ses étudiants, Amin Mahdavi, dont on peut regretter qu'il n'ait pas luimême explicité ses suppositions sur les deux cent seize illustrations qu'aurait comptées initialement le manuscrit avant son démembrement. Bien sûr, les folios des Albums Diez confortent aussi la thèse de Bernard o'Kane sur l'existence d'un grand Shāh nāma djalayride, dont des éléments se retrouvent aussi conservés dans le Hazine 2153 d'Istanbul. La peinture d'époque djalayride est à nouveau évoquée par Massumeh Farhad, qui se penche sur les innovations artistiques de cette époque créative et originale. Enfin, Karine Rührdanz aborde une raison des gestes tout à fait singulière dans un corpus peu étudié du XIV siècle, le Dahnāma, qui survécut aux XV et XVI ${ }^{e}$ siècles, mais qui surtout inspira d'autres corpus moins narratifs.

La dernière partie ("Europe China and Istanbul. The Albums in a Broader Perspective ») opère une ouverture sur les influences étrangères - celles de l'Europe, de la Chine et du monde ottoman - dans les Albums Diez et surtout sur le rôle des peintres étrangers dans la miniature persane. La contribution de Gülru Necipoğlu souligne le rôle des artisans européens, ceux venus de France chez les Mongols par exemple. Elle retrace de façon tout à fait passionnante l'histoire de ces influences occidentales au XIII ${ }^{\mathrm{e}}$ 
et surtout au XIv ${ }^{e}$ siècle, par l'envoi de présents en Orient ou dans le monde ottoman, favorisant l'introduction de thèmes mythiques ou religieux inhabituels dans l'art islamique. L'hypothèse émise que le royaume de Naples (1266-1435) ait pu servir d'intermédiaire entre le monde des ilkhans et le monde européen est intéressante, car cela expliquerait l'existence des huit figures de style européen dans le folio $54 \mathrm{v}$ du H. 2153. À son tour, Ching-Ling Wang analyse quelques peintures et dessins inspirés directement de modèles chinois tant dans les thèmes que dans la composition et l'ordonnance spatiale. Enfin, Serpil Bagci évoque la présence de modèles issus du monde ottoman, soit une typologie curieuse de onze portraits de jeunes hommes questionnant le goût du comte von Diez pour les peintures homo-érotiques.

Les Albums Diez n'ont certes pas livré tous leurs secrets. La personnalité du propriétaire peut en partie expliquer leur composition choisie, en accord avec ses passions d'orientaliste, mais leur raison d'être se trouve aussi dans le regard d'un amateur d'art du $\mathrm{XVIII}^{\mathrm{e}}$ siècle qui présida à leur composition, et dans la tradition orientale de l'album des œuvres de grands maîtres du passé, presque à la façon d'un entomologiste ou d'un botaniste composant un herbier.

Si la fonction de l'album est questionnée et que les illustrations au trait peuvent susciter bon nombre d'explications plausibles sur leur fonction, en revanche, les miniatures sans texte sont plus dérangeantes. On peut y voir des manuscrits démembrés ou martyrisés ou, au contraire, une volonté de sauver des folios épars, détachés, dont on pouvait ignorer la provenance. L'album expose, il montre, il n'explique pas. Parfois, il peut ouvrir une fenêtre sur le passé d'une époque, d'une pratique ; mais plus que tout, il met à l'épreuve les chercheurs qui, habitués à mettre en relation le texte et l'image, oublient parfois que certaines images, même privées de texte, volontairement ou non, peuvent avoir un sens et que certains textes peuvent se lire sans leur image, ou encore que certaines images ont une signification sans existence de texte, ou sans leur texte, comme l'ont montré Emilie Savage-Smith et Yossef Rapoport pour le Livre des curiosités (Oxford, Bodleian Library, Arab or. c 90) . $^{5}$ Dans toute entreprise d'histoire de l'art, le seul véritable écueil n'est pas de faire fausse route, de surinterpréter, mais bien de ne pas retrouver le regard de ceux à qui toute œuvre est primitivement adressée.

\section{NOTES}

1. E. KÜHNEL, « Unbekannte Miniaturen und Zeichnungen aus Berliner Bestinden », dans Akten des

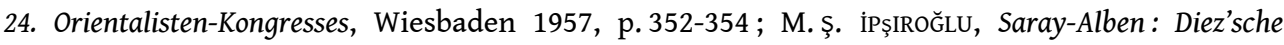
Klebebände aus den Berliner Sammlungen. Beschreibung und stilkritische Anmerkungen, Wiesbaden, 1964.

2. D. J. ROXBURGH, «Heinrich Friedrich Von Diez and His Eponymous Albums: Mss. Dieza. Fols. 70-74 », Muqarnas, 12 (1995), p. 112-136 ; K. RÜHRDANZ, « Illustrationen zu Rašid al-Dins Tarihi Mubärak-i Gäzäni », dans D. AIGLE éd., L'Iran face à la domination mongole, Paris/Téhéran, p. 297-299. 
3. C. ADLE, « Autopsia, in absentia. Sur la date de l'introduction et de la constitution de l'album de Bahrâm Mirzâ par Dust-Mohammad en 951/1544 », Studia Iranica, 19/2 (1990), p. 219-256 ; ID., « Les artistes nommés Dust-Mohammad au XVI e siècle », Studia Iranica, 22/2 (1993), p. 219-296.

4. Celle de Tabriz par exemple, par les Safavides, vit la constitution de l'album de Bahrām Mīrzā b. Shah Esmā'îl, qui est actuellement à Istanbul (Hazine 2154).

5. Y. RAPOPORT, E. SAVAGE-SMITH, Lost Maps of the Caliphs. Drawing the World in Eleventh-Century Cairo, Chicago, 2018.

\section{AUTEURS}

\section{ANNA CAIOZZO}

Université Bordeaux Montaigne, AUSONIUS UMR 5607 